\title{
Real-time capable nonlinear model predictive wheel slip control for combined driving and cornering
}

\author{
Mathias Metzler [0000-0002-3431-6797], Alessandro Scamarcio [0000-0001-5904-8824], Patrick \\ Gruber ${ }^{[0000-0003-1030-6655]}$, and Aldo Sorniotti ${ }^{[0000-0002-4848-058 X]}$ \\ University of Surrey, Guildford, Surrey GU2 7XH, United Kingdom \\ m.metzleresurrey.ac.uk
}

\begin{abstract}
This paper presents a traction controller for combined driving and cornering conditions, based on explicit nonlinear model predictive control. The prediction model includes a nonlinear tire force model using a simplified version of the Pacejka Magic Formula, incorporating the effect of combined longitudinal and lateral slips. Simulations of a front-wheel-drive electric vehicle with multiple motors highlight the benefits of the proposed formulation with respect to a controller with a tire model for pure longitudinal slip. Objective performance indicators provide a performance assessment in traction control scenarios.
\end{abstract}

Keywords: Model predictive control, traction control, combined slip, cornering response, explicit solution.

\section{Introduction}

Extensive literature is available on the topic of wheel slip control, including adaptations for combined driving/braking and cornering conditions. For example, the traction controller (TC) in [1] applies a dynamic saturation of the electric motor torque, by using a friction circle formulation and the estimated lateral tire force. In [2] the longitudinal slip reference is varied as a function of the estimated slip angle. However, the wheel slip control implementations based on model predictive control (MPC), such as [3]-[5], do not include adaptations for combined slip conditions. To the best of our knowledge, the only contribution in this direction is in [6], which proposes an MPC control allocation scheme adopting a linear tire model with varying slip stiffness as a function of slip angle.

This paper presents a TC based on explicit nonlinear model predictive control (eNMPC). The prediction model includes a nonlinear tire force model in the form of a simplified version of the Pacejka Magic Formula, incorporating the effect of combined longitudinal and lateral slips. The benefits of the proposed formulation are highlighted through simulations of traction control scenarios with a front-wheel-drive electric vehicle with in-wheel motors.

In summary, the novelties of this study are:

- The eNMPC based traction controller with a nonlinear tire model for combined slip. 
- The objective assessment of the benefits of the combined slip formulation within the prediction model of the traction controller, with respect to a tire model for pure longitudinal slip.

\section{Optimal control problem formulation}

\subsection{Prediction model}

The prediction model is a half car model or a quarter car model, depending on whether the specific vehicle is two-wheel-drive or four-wheel-drive, with a nonlinear formulation of the steady-state tire force of the driven wheel. The states are the longitudinal component of the vehicle speed in the wheel reference system, $v_{x}$, and the angular wheel speed, $\Omega$. The vehicle body dynamics are described by:

$$
\frac{d v_{x}}{d t}=\frac{1}{m_{H / Q}} F_{x}
$$

where $m_{H / Q}$ is the relevant mass (representing half or a quarter of the vehicle), $t$ is time, and $F_{x}$ is the longitudinal tire force. By neglecting rolling resistance, the wheel dynamics are given by:

$$
\frac{d \Omega}{d t}=\frac{1}{J_{W}}\left(T_{W}-R_{l} F_{x}\right)
$$

where $J_{W}$ is the wheel mass moment of inertia, and $R_{l}$ is the loaded wheel radius. The wheel torque, $T_{w}$, is calculated as:

$$
T_{W}=T_{r e q}-\Delta T
$$

where $T_{r e q}$ is the torque request from a higher level controller (e.g., a drivability controller or a torque-vectoring controller), and $\Delta T$ is the torque reduction imposed by the TC.

The longitudinal tire force in pure longitudinal slip conditions, $F_{x 0}$, is approximated with a simplified version of the Pacejka Magic Formula, with peak factor, $D_{x}$, constant shape factor, $C_{x}$, and stiffness factor, $B_{x}$ :

$$
F_{x 0}=D_{x}\left(F_{z}\right) \sin \left(C_{x} \tan ^{-1}\left(B_{x}\left(F_{z}\right) \kappa_{x}\right)\right)
$$

where $\kappa_{x}$ is the longitudinal slip and $F_{z}$ is the vertical tire load. The peak factor is defined as:

$$
D_{x}=\left(P_{D x 1}+P_{D x 2} d f_{z}\right) \lambda_{\mu} F_{z}
$$

where $P_{D x 1}$ and $P_{D x 2}$ are constants, and the scaling factor $\lambda_{\mu}$ depends on the estimated tire-road friction coefficient. The normalized change in vertical load is:

$$
d f_{z}=\frac{F_{z}-F_{z 0}}{F_{z 0}}
$$

where $F_{z 0}$ is the nominal wheel load. The stiffness factor is defined as: 


$$
B_{x}=\frac{K_{x \kappa}}{C_{x} D_{x}}
$$

with $K_{x \kappa}$ expressed as:

$$
K_{x \kappa}=\left(P_{K x 1}+P_{K x 2} d f_{z}\right) \exp \left(P_{K x 3} d f_{z}\right) F_{z}
$$

where $P_{K x 1}, P_{K x 2}$ and $P_{K x 3}$ are constants.

The influence of combined slip conditions on the longitudinal tire force, $F_{x}$, is modeled through the weighting function $G_{x \alpha}$, with constant shape factor, $C_{x \alpha}$, and variable stiffness factor, $B_{x \alpha}$ :

$$
G_{x \alpha}=\cos \left(C_{x \alpha} \tan ^{-1}\left(B_{x \alpha}\left(\kappa_{x}\right) \alpha\right)\right)
$$

where $\alpha$ is the slip angle. $B_{x \alpha}$ is defined as:

$$
B_{x \alpha}=R_{B x 1} \cos \left(\tan ^{-1}\left(R_{B x 2} \kappa_{x}\right)\right)
$$

with constants $R_{B x 1}$ and $R_{B \times 2}$. The longitudinal tire force in combined slip conditions is given by:

$$
F_{x}=F_{x 0}\left(\kappa_{x}\right) G_{x \alpha}\left(\kappa_{x}, \alpha\right)
$$
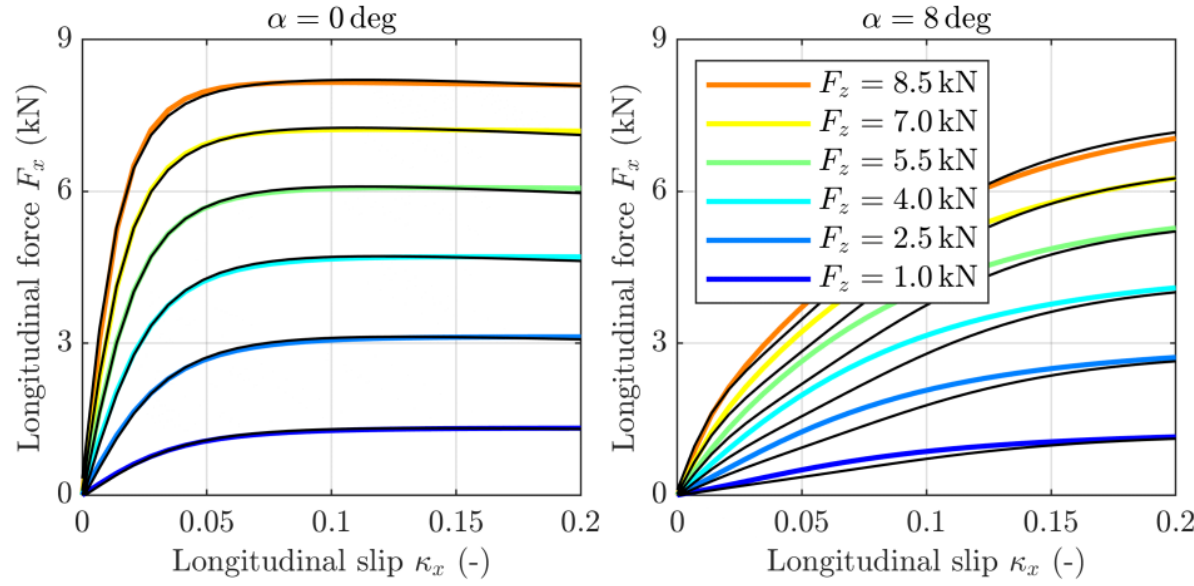

Fig. 1. Longitudinal tire force characteristics as functions of longitudinal slip, for six vertical loads and two slip angles. The black lines indicate the tire forces calculated with the full version of the Pacejka Magic Formula (version 5.2); the colored lines correspond to the tire forces according to the simplified Magic Formula based tire force prediction model.

Fig. 1 reports the longitudinal tire force characteristics as functions of longitudinal slip in high tire-road friction conditions, at six vertical loads (ranging from $1.0 \mathrm{kN}$ to $8.5 \mathrm{kN}$ ), for $\alpha=0 \mathrm{deg}$ and $\alpha=8 \mathrm{deg}$. The graphs are calculated with: i) the full version 5.2 of the Pacejka Magic Formula used in the vehicle simulation model for control system assessment, parametrized with real world tire data (black lines); and ii) the sim- 
plified Pacejka Magic Formula of the TC prediction model, according to (4)-(11) (colored lines). The good match of the results for both slip angle values confirms the accuracy of the simplified tire model formulation.

In traction the longitudinal slip is:

$$
\kappa_{x}=-\frac{v_{s x}}{v_{r}}
$$

with the slip speed, $v_{s x}$, defined as:

$$
v_{s x}=v_{x}-v_{r}
$$

and the linear speed of rolling, $v_{r}$, given by:

$$
v_{r}=\Omega R_{e}
$$

In (14) the effective radius, $R_{e}$, in free rolling conditions is estimated as:

$$
R_{e}=R_{0}-\varrho_{0}\left(D_{R e} \tan ^{-1}\left(B_{R e} \frac{F_{Z}}{F_{z 0}}\right)+F_{R e} \frac{F_{Z}}{F_{z 0}}\right)
$$

where $R_{0}$ is the unloaded wheel radius, $\varrho_{0}$ is the tire deflection at the nominal wheel load, and $D_{R e}, B_{R e}$, and $F_{R e}$ are constant coefficients. The vertical tire deflection, $\varrho$, is defined as the ratio between the vertical tire load, $F_{z}$, and vertical stiffness, $C_{z}$ :

$$
\varrho=\frac{F_{Z}}{C_{z}}
$$

Hence, the loaded wheel radius in (2) is calculated as:

$$
R_{l}=R_{0}-\varrho
$$

\subsection{Cost function and constraints}

The objective of the traction controller is to prevent excessive growth of the longitudinal slip while tracking the torque request, $T_{r e q}$, generated by a higher level controller, and therefore minimizing the wheel torque reduction, $\Delta T$. Hence, the cost function, $V$, of the optimal control problem of this study is defined as:

$$
V=\int_{t_{k}}^{t_{k}+T}\left(r_{u} \Delta T^{2}+q_{s} s^{2}\right) d t
$$

where $t_{k}$ is the current time step, $T$ is the prediction horizon, and $r_{u}$ and $q_{s}$ are tuning weights. The weights include scaling factors to allow equivalent influence in the tuning procedure. The slack variable, $s$, is positive if the actual longitudinal slip, $\kappa_{x}$, exceeds the reference value, $\kappa_{x, r e f}$, in the soft constraint:

$$
\kappa_{x} \leq \kappa_{x, r e f}+s
$$

Based on Eq. (18), an increase of $q_{s}$ improves the slip tracking performance, while an increase of $r_{u}$ reduces the TC torque corrections. Appropriate additional constraints 
are applied, e.g., to ensure that the corrected wheel torque does not exceed the request from the higher level controller:

$$
0 \leq \Delta T \leq T_{\text {req }}
$$

Additionally, the slack variable must be positive semi-definite to only relax and not tighten the soft constraint:

$$
s \geq 0
$$

\subsection{Explicit approach to nonlinear model predictive control}

The resulting nonlinear formulation is parametrized and discretized to solve the optimal control problem. This leads to a multi-parametric nonlinear programming (mp-NLP) problem. An explicit approach (see [5] and [7]) is chosen for a solution implementable in real-time on embedded hardware, i.e., the MPC optimization is solved offline and its solution is stored on the control hardware.

The parameter vector for the mp-NLP problem is $x_{p}=\left[v_{x}, \kappa_{x}, F_{z}, \alpha, T_{r e q}\right]$. The longitudinal slip, $\kappa_{x}$, is selected as parameter, as the resulting formulation scales better in the parameter space exploration than the formulation with the wheel speed, $\Omega$.

The eNMPC algorithm employs multi-parametric quadratic programming (mp-QP) approximations of the mp-NLP problem. The local mp-QP approximations on orthogonal partitions are derived by second order Taylor series expansion of the cost function, and first order Taylor series expansion of the constraint functions. The accuracy is controlled via iterative and recursive partitioning of the parameter space.

The explicit approximate solution of the mp-NLP problem is defined by piece-wise affine functions on polyhedral regions. The online computation reduces to the identification of the polyhedral region for a given parameter vector value, $x_{p}$, and the evaluation of the associated receding horizon feedback law. Therefore, the computationally demanding online solution of the NLP is avoided.

\section{Simulation results}

The results are obtained with a high fidelity MATLAB/Simulink simulation model of a case study front-wheel-drive electric vehicle with in-wheel motors. The tire model of the simulator is based on the version 5.2 of the Pacejka Magic Formula (see Fig. 1). The tire force transients are represented with the realistic relaxation length formulation proposed in [8]. All MPCs are simulated with a prediction model discretization time of $3 \mathrm{~ms}, 5$ prediction steps, 1 control step, and a sampling time of $6 \mathrm{~ms}$.

Fig. 2 reports an example of TC simulation results. The vehicle is initially traveling at a speed of $50 \mathrm{~km} / \mathrm{h}$. At the beginning of the relevant part of the maneuver, a ramp steer is initiated with a steering wheel angle rate of $60 \mathrm{deg} / \mathrm{s}$. During the steering application, a sequence of torque request steps is imposed by the higher level controller. The reference wheel torque is the same on the two driven wheels. The figure compares the results with: i) controller (a), i.e., the eNMPC based traction controller of Section 2, 
with the combined longitudinal and lateral tire force model in (11); and ii) controller (b), i.e., the nonlinear model predictive traction controller with the tire force model in (4), purely based on longitudinal slip. Controller (b) is implemented implicitly, i.e., the solution of the nonlinear model predictive control problem is calculated online at each time step.
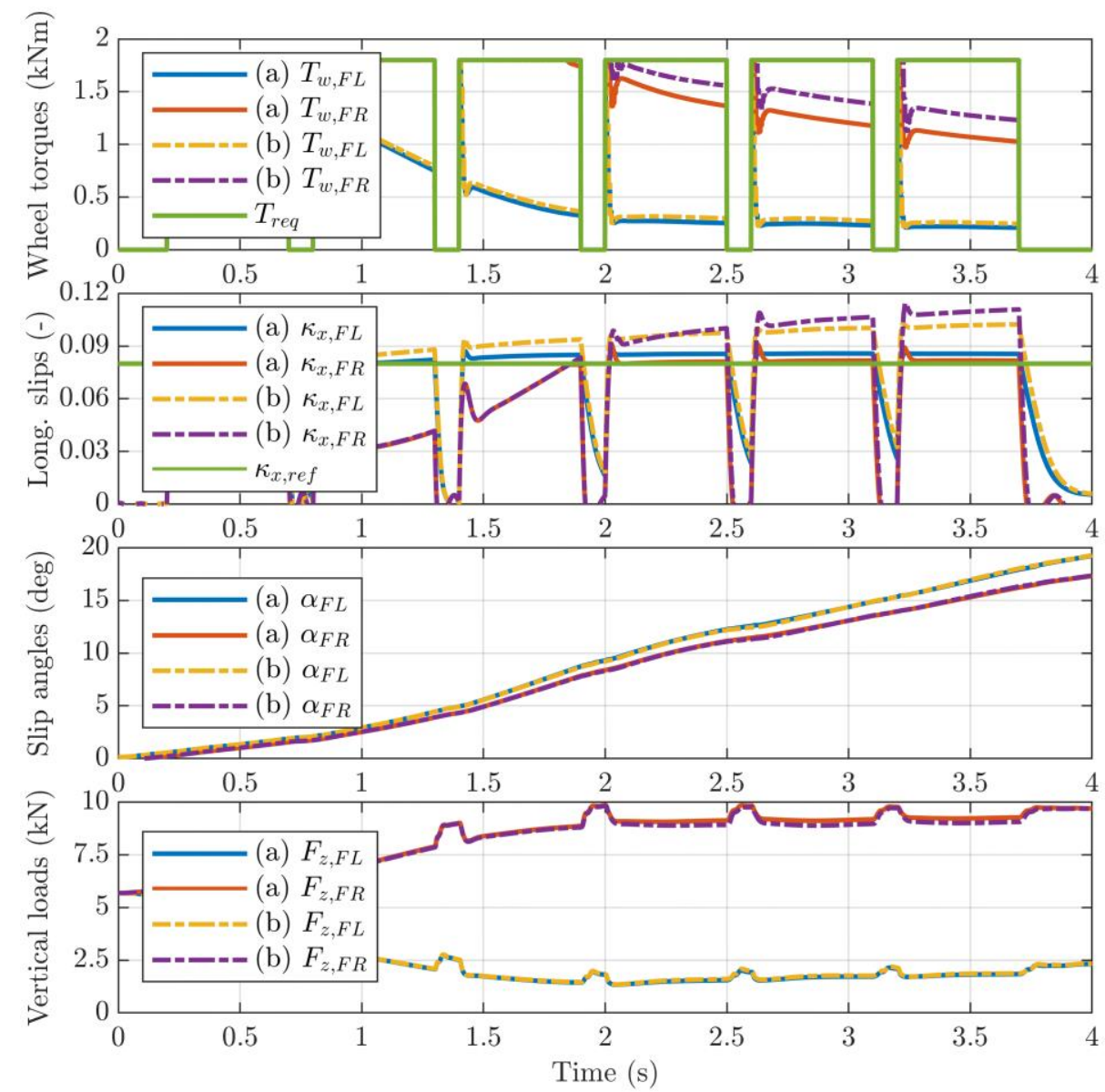

Fig. 2. Time histories of wheel torques, longitudinal slips, tire slip angles, and vertical tire loads, for controllers (a) and (b). The subscripts 'FL' and 'FR' indicate the front left and front right corner.

Controller (a) provides significantly better longitudinal slip tracking performance, especially in conditions of large tire slip angles. This can be observed in Fig. 2 for the last three torque request steps, where the TCs need to apply a torque reduction on both driven wheels. For the first three torque request steps, both traction controllers intervene only on the inner wheel, i.e., the left wheel, because of its lower values of vertical load, which facilitate wheel spinning. The lower levels of longitudinal tire slip with traction 
controller (a) lead to a smaller reduction of the lateral tire forces, and thus result in higher values of vehicle yaw rate, in comparison with controller (b).

To objectively assess the performance of the controllers, the following indicators are introduced:

- $J_{1}$, i.e., the integral of the absolute value of the control error, normalized with time:

$$
J_{1}=\frac{1}{T_{2}-T_{1}} \int_{T_{1}}^{T_{2}}\left|e_{\kappa}\right| d t
$$

where the slip violation, $e_{\kappa}$, is defined as $e_{\kappa}=\kappa_{x}-\kappa_{x, \text { ref }}$ if the longitudinal slip exceeds the slip reference $\kappa_{x, \text { ref }}=0.08$; otherwise $e_{\kappa}$ is set to zero.

- $J_{2}$, i.e., the integral of the absolute value of the control action, normalized with time:

$$
J_{2}=\frac{1}{T_{2}-T_{1}} \int_{T_{1}}^{T_{2}}|\Delta T| d t
$$

$T_{1}$ and $T_{2}$ are the times at the beginning and the end of the relevant part of the simulation, i.e., $T_{1}=0 \mathrm{~s}$ and $T_{2}=4 \mathrm{~s}$ in the specific test. $J_{1}$ and $J_{2}$ are calculated for each front wheel, and then the respective values for the front left and front right corners are summed up to obtain the overall TC indicator.

Table 1 reports the performance indicators for the maneuver of Fig. 2 for: i) the implicit version of controller (a), indicated as iNMPC (a); ii) the explicit version of controller (a), indicated as eNMPC (a); and iii) controller (b), implemented implicitly, and therefore indicated as iNMPC (b).

The enhanced longitudinal slip tracking performance of controller (a) is evident. In fact, for both versions of controller (a) the indicator $J_{1}$ has significantly lower values, by a factor $>4$, with respect to iNMPC (b). This is associated with larger torque reductions for controllers (a), which are reflected in the higher values of $J_{2}$. eNMPC (a) shows marginally inferior performance compared to iNMPC (a), because of the approximations in the generation of the explicit solution. This performance degradation can be tuned - and reduced to a minimum - with appropriate choice of the approximation tolerances for the algorithm generating the explicit solution.

Table 1. Values of the controller performance indicators for the maneuver in Fig. 2.

\begin{tabular}{ccc}
\hline Controller & $J_{1, F L}+J_{1, F R}(-)$ & $J_{2, F L}+J_{2, F R}(\mathrm{Nm})$ \\
\hline iNMPC (a) & $3.3 \mathrm{e}-3$ & 1046 \\
\hline eNMPC (a) & $3.7 \mathrm{e}-3$ & 1044 \\
\hline iNMPC (b) & $17.7 \mathrm{e}-3$ & 946 \\
\hline
\end{tabular}

\section{Conclusion}

This study presented the design, implementation and performance assessment of an eNMPC based traction controller for combined slip conditions. The simulation results demonstrated the influence of tire slip angle on the longitudinal slip tracking perfor- 
mance, which improved by a factor $>4$, according to the defined indicator, for the controllers including a combined slip formulation, with respect to the case of a prediction model considering only longitudinal slip.

Future steps will focus on the revision and extension of the proposed explicit nonlinear model predictive traction controller to meet the practical requirements for its experimental implementation on a vehicle demonstrator with multiple electric motors.

\section{References}

1. Aligia, D. A., Magallan, G. A., De Angelo, C. H.: EV traction control based on nonlinear observers considering longitudinal and lateral tire forces. IEEE Transactions on Intelligent Transportation Systems 19(8), 2558-2571 (2018).

2. Park, J. H., Kim, C. Y.: Wheel slip control in traction control system for vehicle stability. Vehicle System Dynamics 31(4), 263-278 (1999).

3. Borrelli, F., Bemporad, A., Fodor, M., Hrovat, D.: An MPC/hybrid system approach to traction control. IEEE Transactions on Control System Technology 14(3), 541-552 (2006).

4. Satzger, C., de Castro, R.: Combined wheel-slip and torque blending using MPC. In: 2014 International Conference on Connected Vehicles and Expo (ICCVE), 618-624. IEEE, Vienna (2014).

5. Tavernini, D., Metzler, M., Gruber, P., Sorniotti, A.: Explicit nonlinear model predictive control for electric vehicle traction control. IEEE Transactions on Control System Technology 27(4), 1438-1451 (2019).

6. Bächle, T., Graichen, K., Buchholz, M., Dietmayer, K.: Slip-constrained model predictive control allocation for an all-wheel driven electric vehicle. In: $19^{\text {th }}$ IFAC World Congress, IFAC Proceedings Volumes 47(3), 12042-12047 (2014).

7. Metzler, M., Tavernini, D., Sorniotti, A., Gruber, P.: Explicit nonlinear model predictive control for vehicle stability control. In: $9^{\text {th }}$ International Munich Chassis Symposium 2018, 733-752. Springer Vieweg, Wiesbaden (2018).

8. Giangiulio, E., Arosio, D.: New validated tire model to be used for ABS and VDC simulations. In: $3^{\text {rd }}$ International Colloquium on Vehicle-Tire-Road Interaction, 1-17 (2016) 